\title{
ESTUDO DA EVOLUÇÃO DA MATÉRIA ORGÂNICA DO SOLO UTILIZANDO O CARBONO-14 PRODUZIDO NOS ENSAIOS TERMONUCLEARES ATMOSFÉRICOS
}

\author{
J. M. FLEXOR*, P. F. S. LÔBO* e J. L. RAPAIRE**
}

\begin{abstract}
The specific activities $\left({ }^{14} \mathrm{C} /{ }^{12} \mathrm{C}\right)$ of the free fulvic acids (FFA), humic acids (HA) and humine $(\mathrm{H})$ of the $0-5 \mathrm{~cm}$ layer of ferralitic florestal soil of Bahia exhibited: $\delta^{14} \mathrm{C}(\mathrm{FFA})=$ $=(15 \pm 1) \% ; \delta^{14} \mathrm{C}(\mathrm{HA})=(25 \pm 1) \%$ and $\delta^{14} \mathrm{C}(\mathrm{H})=(36 \pm 1) \%$. Laws of conservation regarding the evolution of the specific activity of atmospheric $\mathrm{CO}_{2}$ (for $13^{\circ} \mathrm{S}$ latitude), applied to a simple model of fractionation of organic matter, allowed to the estimation of the formation rates for the three fractions $(0-5 \mathrm{~cm}) q_{\mathrm{FFA}}=0.005 \mathrm{~kg} / \mathrm{m}^{2} ; q_{\mathrm{HA}}=0.037 \mathrm{~kg} / \mathrm{m}^{2}$ and $q_{\mathrm{H}}=0.180 \mathrm{~kg} / \mathrm{m}^{2}$. Simplified hypotheses lead to mean residence times, for the $(0-5 \mathrm{~cm})$ layer, of $T_{R}^{\mathrm{FFA}}=7$ years, $T_{R}^{\mathrm{AH}}=15$ years, and $T_{R}^{\mathrm{H}}=34$ years. The obtained sequence for the above residence times agrees with the generally admitted hypotheses.
\end{abstract}

INTRODUÇÃo Os métodos químicos de extração que definem as diferentes frações do húmus do solo fornecem somente algumas indicações sobre suas estabilidades. Algumas hipóteses relacionam comumente estabilidade com poder extrativo dos reativos utilizados: os ácidos fúlvicos livres, primeira fração extraída, corresponderiam à fração mais jovem, enquanto que a humina, fração que subsiste às extrações, seria a mais estável (Duchaufour, 1965; Aubert et al., 1967).

Experiências visando obter informações sobre o comportamento das frações orgânicas foram realizadas no laboratório, utilizando-se moléculas marcadas com ${ }^{14} \mathrm{C}$, como traçador (Scharpenseel et al., 1962). Os resultados obtidos dão indicações sobre as taxas de incorporação, durante um dado período de incubação, de ${ }^{14} \mathrm{C}$ pelas diferentes frações. Nada se pode concluir sobre estabilidade das frações: nas experiências efetuadas in vitro, as moléculas marcadas são introduzidas diretamente na amostra de solo enquanto que, na natureza, o carbono é incorporado a partir do $\mathrm{CO}_{2}$ atmosférico através da fotossíntese; uão é possível, no laboratório, reproduzir todos os parâmetros que prevalecem na natureza: condições climáticas, físico-químicas, atividade biológica, etc.

As dificuldades encontradas na interpretação dessas experiências para estudos da formação das várias frações levaram alguns autores a utilizar o ${ }^{14} \mathrm{C}$ natural, datando frações orgânicas de solos antigos.

$\mathrm{O}{ }^{14} \mathrm{C}$, nuclídeo radioativo $\left(T_{1 / 2}=5730\right.$ anos) de origem cosmogênica, formado na alta atmosfera, é rapidamente oxidado e misturado ao $\mathrm{CO}_{2}$ atmosférico. Marca, assim, as diferentes etapas de absorção e trocas envolvidas no ciclo do carbono. O prevalecimento de um estado de equilíbrio entre taxa de produção e taxa de decaimento permitiu estabelecer um método de datação bem conhecido (Libby, 1952).

Scharpenseel et al. (1970) realizaram um grande número de experiências sobre frações orgânicas de vários tipos de solos antigos. Para um chernozem, o carbono total e os ácidos húmicos mostraram a mesma idade dentro dos limites dos erros experimentais; para um podzol, as idades obtidas para várias frações não são muito diferentes, $2828 \pm 60$ anos em média. A importância do erro estatístico (devida somente ao grande valor da meia-vida Bahia

* Laboratório de Fracas Radioatividades do Instituto de Física da Universidade Federal da **Centre Scientifique de Mônaco, Principado de Mônaco 
do ${ }^{14} \mathrm{C}$ ) e a constância da atividade específica do $\mathrm{CO}_{2}$ atmosférico, pelo menos durante os últimos 10000 anos, sem perturbações apreciáveis (Damon et al., 1965; Stuiver, 1961), não permitem seguir processos dinâmicos de períodos curtos, quando comparados com a meia-vida do ${ }^{14} \mathrm{C}$.

Em 1954, início dos testes nucleares em grande escala, realizados principalmente no Hemisfério Norte, foi rompido o equilíbrio das atividades específicas existentes entre os diversos reservatórios que participam do ciclo do carbono. Seguiu-se uma evolução diferente para cada reservatório que serviu de base para inúmeros trabalhos envolvendo trocas rápidas do carbono entre a estratosfera e troposfera, camadas superficiais e profundas dos oceanos, etc. (Rafter et al., 1965; Rafter, 1968; Nydal, 1968). Propõe-se, aqui, desenvolver um estudo semelhante sobre as diferentes frações da matéria orgânica do solo. Este artigo apresenta os primeiros resultados.

Evolução da atividade específica do $\mathrm{CO}_{2}$ atmosférico Desde que as diversas frações da matéria orgânica provêm direta ou indiretamente da decomposição da matéria orgânica fresca (folhas, sistemas radiculares, ramos, etc.), é preciso conhecer a evolução da atividade específica do seu carbono. Nakhla (1968) mostrou que era idêntica à do $\mathrm{CO}_{2}$ do ar, não apresentando variações brutais devidas à direção dos ventos, estações climáticas anuais, proximidade de zonas industriais onde há combustão de carbono fóssil, etc.

A evolução da atividade específica do ar, em função da latitude e do tempo, foi objeto de pesquisas realizadas por numerosos laboratórios, sobretudo a partir de 1954, quando se iniciaram os ensaios termonucleares em grande escala. Pode-se constatar que medidas realizadas para $\lambda=20^{\circ} \mathrm{S}, 26^{\circ} \mathrm{S}$ e $41^{\circ} \mathrm{S}$ não apresentam diferenças significativas e que, a partir de 1968, a evolução é única para os dois hemisférios (Nydal, 1963; Rafter et al., 1957; Münnich et al., 1958; Münnich et al., 1962; Nydal, 1968, e Vogel, 1971).

A extrapolação feita sobre a curva única (1968-1970), cujos últimos dados na literatura vão somente até o início de 1970 (Vogel, 1971), ajusta-se, dentro dos erros experimentais, com o valor da atividade do $\mathrm{CO}_{2}$ atmosférico em fevereiro de 1971, medida em Salvador (Ba) $\left(\lambda=13^{\circ} \mathrm{S}\right), \delta^{14} \mathrm{C}=(55 \pm 1) \% *$. Decorre que a curva mostrada na Fig. 1, que abrange o período 1954-1971, pode ser considerada como representativa, a menos de $2 \%$, da variação da atividade específica do $\mathrm{CO}_{2}$ atmosférico para $\lambda=13^{\circ} \mathrm{S}$.

Escolha do solo para estudo CARACTERISTICAS Até o presente foram realizados poucos trabalhos visando o estudo da evolução da matéria orgânica a partir de medidas de atividade específica de origem termonuclear. Todos os resultados, pelo menos ao nosso conhecimento, referem-se a solos de zonas temperadas do Hemisfério Norte. Deve ser interessante, para fins de comparações ulteriores, efetuar um estudo semelhante em Zona Tropical Úmida, onde a evolução da matéria orgânica é diferente, dependendo principalmente da

a) temperatura média alta, praticamente constante, implicando no crescimento contínuo dos vegetais, sem variações apreciáveis;

b) ação biológica teoricamente mais intensa e constante do que em zonas temperadas;

c) pluviosidade importante, que, associada ao fator temperatura, deveria acentuar o fenômeno da biodegradação, pelo menos para solos bem drenados e pouco ácidos.

$* \delta^{14} \mathrm{C}(\%)=\left\{\left({ }^{14} \mathrm{C} /{ }^{12} \mathrm{C}\right)_{a}-\left({ }^{14} \mathrm{C} /{ }^{12} \mathrm{C}\right)_{p}\right\} /\left({ }^{14} \mathrm{C} /{ }^{12} \mathrm{C}\right)_{p}$, onde $a=$ amostra, $p=$ padrão $(1950)$. $\delta^{14} \mathrm{C}(1950)=0$, por definição 


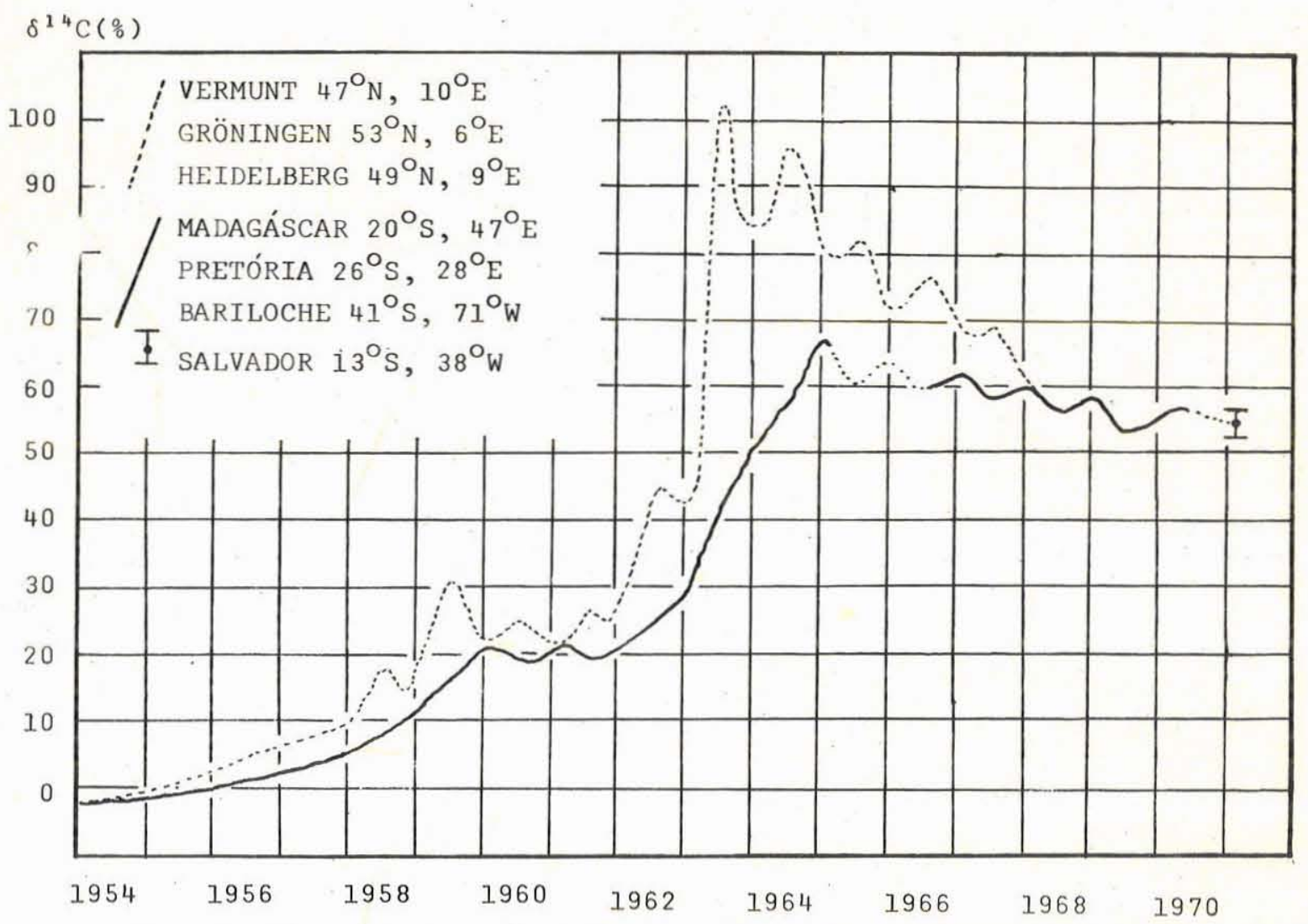

Figura 1 - Curva de evolução da atividade específica do $\mathrm{CO}_{2}$ atmosférico (Vogel. 1971)

Para este primeiro estudo, escolheu-se um solo florestal situado em Ondina, Saliador $\left(12^{\circ} 55^{\prime} \mathrm{S}, 38^{\circ} 31^{\prime} \mathrm{W}\right)$. Trata-se de um solo ferralítico, areno-argiloso, proveniente da alte1 ação do cristalino, de cobertura vegetal de densidade média e próximo ao mar. A pluviosidade e temperatura médias são, respectivamente, $1700 \mathrm{~mm} /$ ano e $25,1^{\circ} \mathrm{C}$. As características do perfil amostrado são apresentadas na Tab. I.

Extração e dosagem do carbono das frações orgânicas A amostra, inicialmente seca ao ar, é peneirada $(<0,5 \mathrm{~mm})$ a fim de eliminar detritos vegetais cuja estrutura ainda seja organizada.

A dosagem do carbono total é feita por via úmida, método Anne (1945), onde o carbono é oxidado por uma mistura de bicromato de potássio e ácido sulfúrico. O excesso de bicromato é titulado por uma solução de sal de Mohr. Os valores encontrados estão especificados na Tab. I.

O método de extração das diversas frações orgânicas adotado neste trabalho foi idealizado por B. Dabin (1971). As etapas de extração estão representadas no Esquema I.

A primeira extração $\left(\mathrm{H}_{3} \mathrm{PO}_{4} 2 \mathrm{~N}\right)$ é um pré-tratamento onde são eliminadas, por filtração, as matérias leves em via de humificação que levariam à neoformação de frações mais evoluídas durante as etapas seguintes, pela ação dos reativos alcalinos sobre a matéria fresca. Dessa maneira eliminam-se efeitos indesejáveis que poderiam ocorrer durante o processo de extração - resultados quantitativos falsos para as frações obtidas - e a conseqüente contaminação com carbono de atividade específica atual. 
Tabela I

\begin{tabular}{llcl}
\hline $\begin{array}{c}\text { Composição granulométrica }(\%) \\
\text { (dispersão em NaOH } 0,1 \mathrm{~N})\end{array}$ & $0-5$ & $5-15$ & $15-45$ \\
\hline \hline areia grossa $(2-0,2 \mathrm{~mm})$ & 28,8 & 23,4 & 18,4 \\
\hline areia fina $(0,2-0,05 \mathrm{~mm})$ & 14,6 & 13,8 & 10,1 \\
\hline silte $(0,05-0,002 \mathrm{~mm})$ & 33,5 & 26,6 & 28,6 \\
\hline "argila” $(<0,002 \mathrm{~mm})$ & 20,1 & 36,2 & 42,9 \\
\hline $\begin{array}{l}\text { argila natural }(\mathrm{kaolinita}, \\
\text { difração de raios X) }\end{array}$ & 10,4 & 42,9 & 27,2 \\
\hline Características quimicas & & & \\
\hline $\mathrm{pH}$ & 6,2 & 6,4 & 6,3 \\
\hline $\mathrm{Al}(\mathrm{meq} / 100 \mathrm{~g})$ & 0,0 & 0,0 & 0,0 \\
\hline $\mathrm{Ca}(\mathrm{meq} / 100 \mathrm{~g})$ & 7,0 & 2,5 & 1,4 \\
\hline $\mathrm{Mg}(\mathrm{meq} / 100 \mathrm{~g})$ & 4,0 & 3,7 & 3,1 \\
\hline $\mathrm{K}(\mathrm{meq} / 100 \mathrm{~g})$ & 0,32 & 0,24 & 0,14 \\
\hline $\mathrm{P}$ (ppm) & 3 & 1 & 1 \\
\hline $\mathrm{C}$ total $(\%)$ & 2,80 & 2,30 & 1,46 \\
\hline $\mathrm{Matéria} \mathrm{orgânica} \mathrm{total}(\%)$ & 4,83 & 3,97 & 2,52 \\
\hline
\end{tabular}

Esquema I

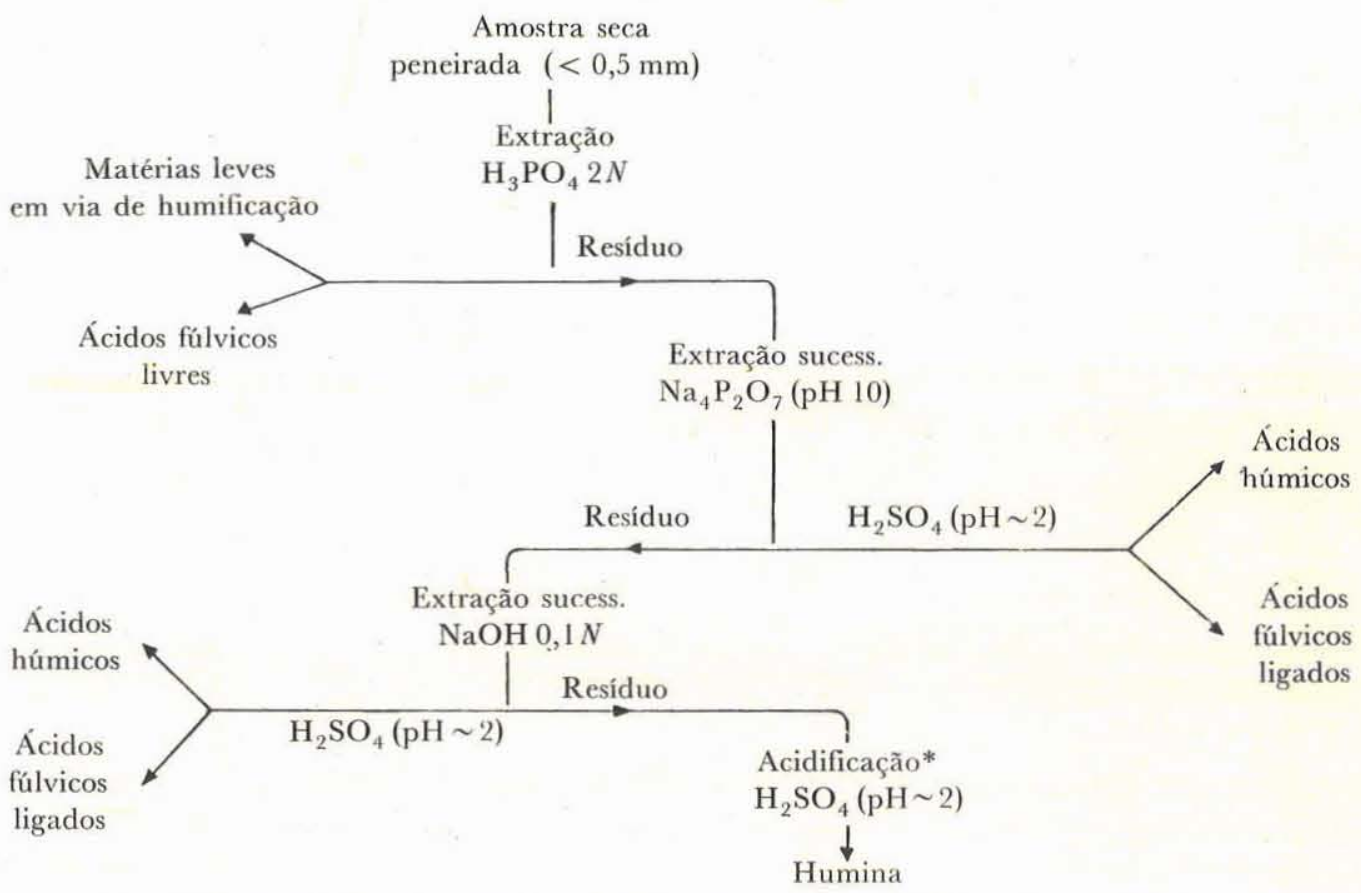

*A acidificação da humina, resíduo da última extração $\mathrm{NaOH}$, é aqui introduzida para evitar a contaminação com $\mathrm{CO}_{2}$ atmosférico 
As extrações são efetuadas sobre $1 \mathrm{~kg}$ de amostra, em média, para que se tenha carbono suficiente, por fração, para poder medir a atividade específica sob a forma de $\mathrm{CO}_{2}$ muito puro. Tomam-se alíquotas conhecidas de cada fração e faz-se a respectiva dosagem de carbono. Os resultados obtidos são apresentados na Tab. II.

Tabela II

\begin{tabular}{cccccc}
\hline $\begin{array}{c}\text { Lamada } \\
(\mathrm{cm})\end{array}$ & $\begin{array}{c}\text { Ácidos } \\
\text { fúlvicos } \\
\text { livres } \\
\mathrm{C} \%\end{array}$ & $\begin{array}{c}\text { Ácidos } \\
\text { húmicos } \\
\left(\mathrm{Na}_{4} \mathrm{P}_{2} \mathrm{O}_{7}\right) \\
\mathrm{C} \%\end{array}$ & $\begin{array}{c}\text { Ácidos } \\
\text { fúlvicos } \\
\text { ligados } \\
\left(\mathrm{Na}_{4} \mathrm{P}_{2} \mathrm{O}_{7}\right) \\
\mathrm{C} \%\end{array}$ & $\begin{array}{c}\text { Ácidos húmicos } \\
+ \\
\text { fúlvicos ligados } \\
(\mathrm{NaOH}) \\
\mathrm{C} \%\end{array}$ & $\begin{array}{c}\text { Humina } \\
\mathrm{C} \%\end{array}$ \\
\hline \hline $0-5$ & 0,13 & 0,31 & 0,27 & 0,30 & 1,63 \\
\hline $15-45$ & 0,22 & 0,16 & 0,40 & 0,12 & 0,41 \\
\hline
\end{tabular}

A instalação de contagem $\left({ }^{14} \mathrm{C} /{ }^{12} \mathrm{C}\right)$ utilizada para as medidas tem as seguintes características:

a) contador proporcional central, volume útil =1,2 litros;

b) gás de enchimento: $\mathrm{CO}_{2}$ muito puro a 1 atmosfera;

c) ruído de fundo $=2,3$ contagens por minuto;

d) atividade do padrão $\mathrm{NBS}=6,1$ desintegrações por minuto;

e) idade máxima teoricamente detectável $=33000$ anos.

Medidas obtidas INTERPRETAÇÃO E RESULTADOS Medidas efetuadas sobre ácidos fúlvicos livres (AFL), ácidos húmicos $(\mathrm{AH})$ e humina $(\mathrm{H})$ deram:

$$
\begin{aligned}
\text { camada } & 0-5 \mathrm{~cm} \\
\delta^{14} \mathrm{C}(\mathrm{AFL}) & =(15 \pm 1) \% \\
\delta^{14} \mathrm{C}(\mathrm{AH}) & =(25 \pm 1) \% \\
\delta^{14} \mathrm{C}(\mathrm{H}) & =(36 \pm 1) \% .
\end{aligned}
$$

Estas medidas fornecem as "idades aparentes" (I.A.) das frações acima, definidas wino o intervalo de tempo entre o ano de amostragem (1971) e anos correspondentes às atividades medidas, na curva de evolução $F(T)$ (Fig. 1):

$$
\text { camada } \mathrm{U}-\mathrm{J} \mathrm{cm}
$$

$$
\begin{aligned}
& \text { I.A. (AFL) }=13 \pm 1 \text { anos } \\
& \text { I.A. (AH) }=9 \pm 1 \text { anos } \\
& \text { I.A. (H) }=8 \pm 1 \text { anos. }
\end{aligned}
$$

Vemos que, para a camada $0-5 \mathrm{~cm}$, os AFL seriam mais "velhos" do que os AH e a $\mathrm{H}$. Na realidade, as atividades medidas representam o resultado de "contaminações" sucessivas anuais que provêm direta ou indiretamente da atividade específica da matéria fresca morta nos anos precedentes.

Supondo que o solo estudado esteja em equilíbrio com relação ao seu ciclo do carbono, podemos aplicar leis de conservação envolvendo as contribuições da matéria fresca e as frações estudadas. Neste primeiro trabalho suporemos um modelo de formação no qual a matéria orgânica fresca contribua de maneira direta e independente para cada fração. Nessas condições, as leis de conservação para uma fração $i(i=\mathrm{AFL}, \mathrm{AH}, \mathrm{H})$ nos permitem 
escrever:

onde:

$$
A_{i}^{E}(T)=\frac{1}{Q_{i}}\left\{A_{i}^{E}(T-1)\left(Q_{i}-q_{i}\right)+\frac{q_{i}}{T_{F}^{i}} \sum_{\substack{T_{n}=T+1-T^{i} \\ F}}^{I_{n}=T} F\left(T_{n}\right)\right\}
$$

$A_{i}^{E}(T), \quad$ é atividade específica da fração $i$ no ano $T$;

$A_{i}^{E}(T-1)$, atividade específica da fração $i$ no ano precedente;

$F(T), \quad$ atividade específica da matéria fresca no ano $T$;

$Q_{i}, \quad$ quantidade de carbono na fração $i$ num horizonte qualquer;

$q_{i}$, quantidade de carbono da fração $i$ introduzida anualmente no mesmo horizonte;

$T_{F}^{i}$, tempo de formação de $q_{i}$ a partir da matéria fresca. Supõe-se aqui que, para uma quantidade dada de matéria vegetal morta no ano $T$, a taxa inicial de produção da fração $i$ permaneça constante durante todo o tempo de formação.

Se definirmos tempo de residência como a relação entre a quantidade presente num horizonte qualquer $\left(Q_{i}\right)$ e aquela fornecida a partir da decomposição da matéria fresca $\left(q_{i}\right)$, isto é, $T_{R}^{i}=\frac{Q_{i}}{q_{i}}$ e fazendo, arbitrariamente, $q_{i}=1$ em (1), virá

$$
A_{i}^{E}(T)=\frac{1}{T_{R}^{i}}\left\{\frac{1}{T_{F}^{i}} \sum_{\substack{T_{n}=T+1-T^{i} \\ F}}^{T_{n}=T} F\left(T_{n}\right)+A_{i}^{E}(T-1)\left(T_{R}^{i}-1\right)\right\}
$$

A curva de evolução $F(T)$ mostra que a partir de 1955 os ensaios termonucleares, realizados nos anos anteriores, começam a perturbar o estado de equilíbrio que prevalecia até o ano anterior, quando então $\delta^{14} \mathrm{C}=-2 \%$ ("Efeito Suess", Revelle et al., 1957). A hipótese de equilíbrio implicando $A_{i}^{E}(T \leqq 1954)=F(T \leqq 1954)=-2 \%$, podemos, utilizando a relação (2), construir as curvas de evolução da atividade específica para a fração $i, A_{i}^{E}\left(T_{F}^{i}, T_{R}^{i}, T\right)$ para $1955 \leqq T \leqq 1971$. As relações $T_{F}^{i}-T_{R}^{i}$ compatíveis com as atividades medidas estão explicitadas nas Figs. 2, 3 e 4, respectivamente.

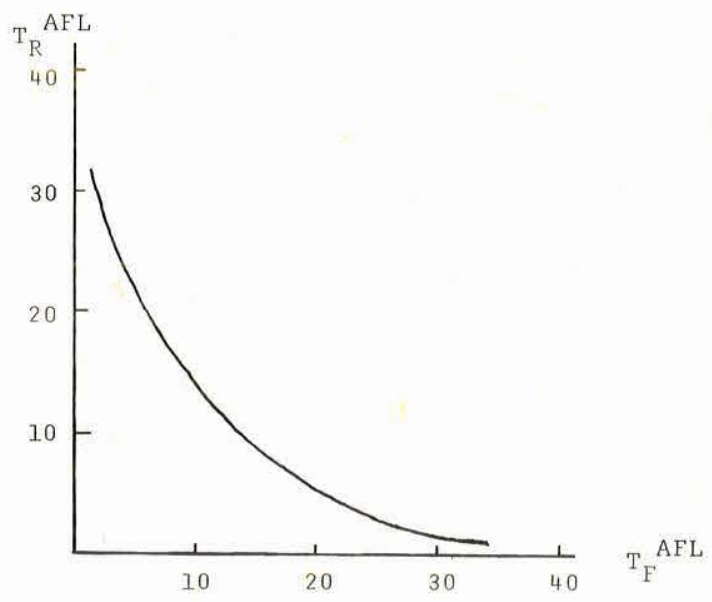

Figura $2-A_{\mathrm{AFL}}^{E}\left(T_{F}^{\mathrm{AFL}}, T_{R}^{\mathrm{AFL}}, 1971\right)=15 \pm 1 \%$ 


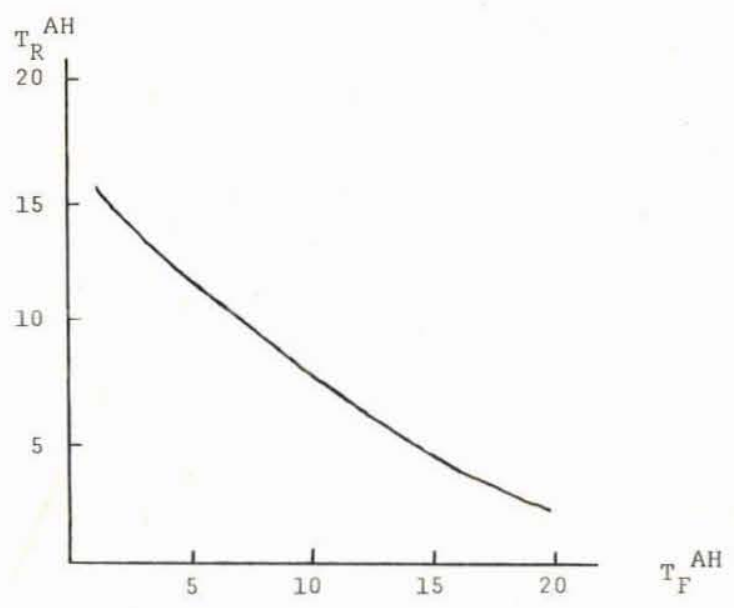

Figura $3-A_{\mathrm{AH}}^{E}\left(T_{\boldsymbol{F}}^{\mathrm{AH}}, T_{\boldsymbol{R}}^{\mathrm{AH}}, 1971\right)=25 \pm 1 \%$

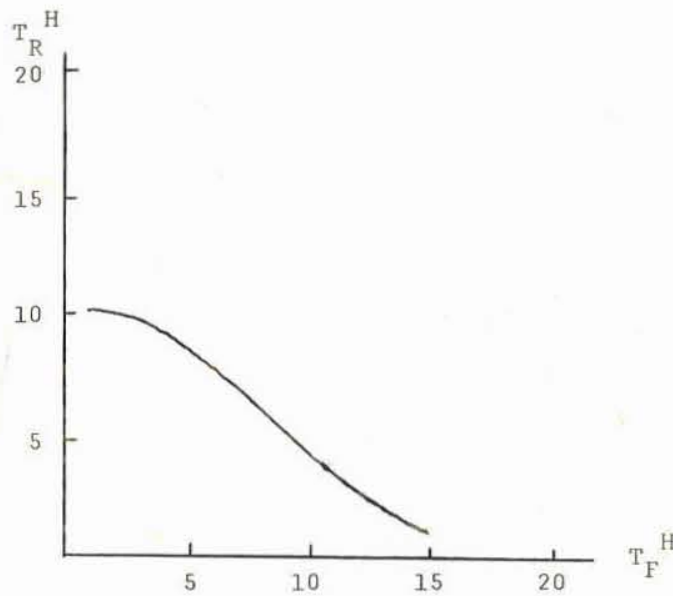

Figura $4-A_{\mathrm{H}}^{E}\left(T_{F}^{\mathrm{H}}, T_{R}^{\mathrm{H}}, 1971\right)=36 \pm 1 \%$

Se limitarmos superiormente os $T_{F}^{i}$ por 4 anos (Siefferman, 1971), obteremos os campos de variação correspondentes para os $T_{R}^{i}: 25 \leqq T_{R}^{\mathrm{AFL}} \leqq 31$ anos, $14 \leqq T_{R}^{\mathrm{AH}} \leqq 16$ anos, $9 \leqq T_{R}^{\mathrm{H}} \leqq 10$ anos, ou seja, em média:

$$
T_{R}^{\mathrm{AFL}}=28 \text { anos, } \quad T_{R}^{\mathrm{AH}}=15 \text { anos, } \quad T_{R}^{\mathrm{H}}=10 \mathrm{anos},
$$

ou, pela definição de $T_{\mathrm{R}}^{i}\left(=Q_{i} / q_{i}\right)$, segue:

$$
\frac{Q_{\mathrm{AFL}}}{q_{\mathrm{AFL}}}=28 \operatorname{anos}, \quad \frac{Q_{\mathrm{AH}}}{q_{\mathrm{AH}}}=15 \text { anos, } \quad \frac{Q_{\mathrm{H}}}{q_{\mathrm{H}}}=10 \text { anos. }
$$

Estes resultados equivalem a dizer que a relação entre as quantidades de fração $i$ presentes no solo e aquelas fornecidas anualmente pela decomposição da matéria fresca são, respectivamente, 28 para os AFL, 15 para os $\mathrm{AH}$ e 10 para a $\mathrm{H}$. Nada podemos dizer, por enquanto, a respeito dos valores de $q_{i}$, mas as dosagens de carbono de todas as frações obtidas permitem estimar, para o horizonte $0-5 \mathrm{~cm}$, as porcentagens de carbono das frações 
em estudo: $\mathrm{C}(\mathrm{AFL})=0,13 \%, \mathrm{C}(\mathrm{AH})=0,48 \%$ e $\mathrm{C}(\mathrm{H})=1,63 \%$. Para obter estes resultados, assumimos que, para o solo em estudo, $\mathrm{AH} / \mathrm{AF}\left(\mathrm{Na}_{4} \mathrm{P}_{2} \mathrm{O}_{7} \mathrm{pH} 10\right)=\mathrm{AH} / \mathrm{AF}$ ( $\mathrm{NaOH} 0,1 \mathrm{~N})$ (Thomann, 1963), onde AF são os ácidos fúlvicos ligados. Assumindo, para um solo ferralítico, uma densidade média de $2,3 \mathrm{~g} / \mathrm{cm}^{3}$, obtemos a massa de carbono, para o horizonte $0-5 \mathrm{~cm}$, das frações:

$$
Q_{\mathrm{AFL}}=0,15 \mathrm{~kg} \mathrm{C} / \mathrm{m}^{2}, \quad Q_{\mathrm{AH}}=0,55 \mathrm{~kg} \mathrm{C} / \mathrm{m}^{2}, \quad Q_{\mathrm{H}}=1,89 \mathrm{~kg} \mathrm{C} / \mathrm{m}^{2} .
$$

Podemos agora, a partir dos resultados acima, deduzir as quantidades de carbono introduzidas anualmente sob a forma de fração $i\left({ }^{q}{ }_{i}\right)$ para a camada $0-5 \mathrm{~cm}$ :

$$
\begin{aligned}
& q_{\mathrm{AFL}}=0,005 \mathrm{~kg} / \mathrm{m}^{2} / \text { ano, } \\
& q_{\mathrm{AH}}=0,037 \mathrm{~kg} / \mathrm{m}^{2} / \text { ano, } \\
& q_{\mathrm{H}}=0,180 \mathrm{~kg} / \mathrm{m}^{2} / \text { ano. }
\end{aligned}
$$

Se quisermos comparar os tempos de residência das frações, será preciso introduzir uma normalização que nos leve a determinar espessuras de solo onde $Q_{\mathrm{AFL}}=Q_{\mathrm{AH}}=Q_{\mathrm{H}}$, respectivamente. Esta normalização é feita tomando como espessura de referência o horizonte $0-5 \mathrm{~cm}$ para os $\mathrm{AH}$, na qual $Q_{\mathrm{AH}}=0,55 \mathrm{~kg} \mathrm{C} / \mathrm{m}^{2}$ e assumindo que a concentração de carbono, para os AFL na camada $0-5 \mathrm{~cm}(0,13 \%)$, é uma média representativa para todo o solo. As espessuras assim calculadas, correspondendo a uma quantidade de $0,55 \mathrm{~kg} \mathrm{C} / \mathrm{m}^{2}$, são:

isto é,

$$
\begin{aligned}
& \mathrm{AFL} \longrightarrow 19,0 \mathrm{~cm}, \\
& \mathrm{AH} \longrightarrow 5,0 \mathrm{~cm} \text { (referência), } \\
& \mathrm{H} \longrightarrow 1,5 \mathrm{~cm},
\end{aligned}
$$

$$
\begin{aligned}
& T_{\mathrm{R}}^{\mathrm{AFL}}=28 \text { anos } \longrightarrow 19,0 \mathrm{~cm}, \\
& T_{R}^{\mathrm{AH}}=15 \text { anos } \longrightarrow 5,0 \mathrm{~cm} \text { (referência), } \\
& T_{R}^{\mathrm{H}}=10 \text { anos } \longrightarrow 1,5 \mathrm{~cm} .
\end{aligned}
$$

Supondo que a introdução, no horizonte $0-19 \mathrm{~cm}$, dos ácidos fúlvicos livres formados a partir da matéria fresca, é realizada em sua totalidade nos cinco primeiros $\mathrm{cm}$ de solo e que toda a humina presente nesta camada provém dos dois primeiros $\mathrm{cm}$, a normalização à espessura de referência conduz a

$$
\begin{aligned}
& T_{R}^{\mathrm{AFL}}(0-5 \mathrm{~cm})=7 \text { anos, } \\
& T_{R}^{\mathrm{AH}}(0-5 \mathrm{~cm})=15 \text { anos, } \\
& T_{R}^{\mathrm{H}}(0-5 \mathrm{~cm})=34 \text { anos. }
\end{aligned}
$$

DISCUSSÃO E CONCLUSÕES Todos os resultados obtidos baseiam-se na validez do modelo utilizado, onde os ácidos fúlvicos livres, os ácidos húmicos e a humina proviriam direta e independentemente da matéria fresca. Esse modelo, demasiado simples, talvez só corresponda vagamente aos processos reais de fracionamento da matéria orgânica. Permitiu-nos, entretanto, estimar as quantidades de carbono introduzido anualmente sob a forma das frações estudadas, e a sucessão $T_{R}^{\mathrm{AFL}}<T_{R}^{\mathrm{AH}}<T_{R}^{\mathrm{H}}$ encontrada para os tempos de residência está de acordo com a hipótese geralmente aceita, segundo a qual uma fração será tanto mais estável, do ponto de vista de biodegradação e mobilidade, quanto mais ligada for às argilas.

Por outro lado, o estudo de um modelo simples, para o qual os ácidos húmicos e a humina proviriam de contribuições em proporções variáveis da matéria fresca e dos ácidos fúlvicos livres, conduziu a limites de variação $\operatorname{dos} T_{R}^{i}$ em função $\operatorname{dos} T_{F}^{i}$, sempre inferiores àqueles determinados pelo modelo utilizado. Verificou-se também que a contribuição mí- 
nima de matéria fresca para a formação dos ácidos húmicos e da humina seria, respectivamente, de 60 e $75 \%$, o que nos leva a admitir a validade dos resultados obtidos a menos de um fator 2 .

Estendendo as medidas de atividade específica a todos os horizontes e a outras frações, talvez menos representativas da evolução da matéria orgânica de um solo, e sobretudo continuando as medidas nos próximos anos, será possível estudar modelos mais complexos, mais próximos da realidade.

Concluindo, o método do carbono-14 aplicado ao estudo dos solos e, de um modo mais geral, às técnicas nucleares utilizadas como complemento aos métodos clássicos da ciência dos solos deveriam permitir abordar de maneira mais fácil, e provavelmente mais eficiente, problemas diversos, tais como a renovação da cobertura vegetal de um solo florestal, tempos de residência e tempos de formação para as diversas frações orgânicas, mineralização, degradação, erosão. Esta lista não é exaustiva.

Agradecimentos É com prazer que agradecemos às entidades que, financiando equipamentos e pessoal altamente especializado, permitiram a montagem do Laboratório de Fracas Radioatividades da UFBa, a serem: CNPq, CAPES, UNESCO (Projeto BRA-4), Ministério do Planejamento, SUDENE e CNEN. Estendemos os agradecimentos ao Departamento 01 do Instituto de Geociências da UFBa, que nos propiciou a oportunidade de contar com a ajuda de especialistas da O.R.S.T.O.M. Dr. G. Siefferman, que, desde o começo deste trabalho, guiou-nos na escolha de solos de interesse, participando sempre de frutuosas discussões, e Dr. B. Dabin, que nos ajudou a desenvolver a técnica de extração das frações da matéria orgânica. Finalmente, os nossos agradecimentos à CEPLAC, que efetuou as dosagens das bases trocáveis e a granulometria do perfil.

\section{BIBLIOGRAFIA}

ANNE - 1945 - Dosage rapide du carbone organique des sols. Ann. Agr. 2, pp. 161-172

AUBERT, G. e BOUlAine, J. - 1967 - La Pédologie, 1.a ed., Paris, Press Universitaires de France, $126 \mathrm{pp}$.

DABIN, B. - 1971 - Étude d'une méthode d'extraction de la matière humique du sol. "Science du Sol" 1, Suppl. Ass. Fr. Ét. Sol, pp. 47-63

DAMON, P. E., LONG, A. e GRAY, D. C. - 1965 - Fluctuations of atmospheric carbon-14 during the last six millenia. Proc. 6th Conf. Radiocarbon Tritium Dating, Pullman, Washington. U.S.A.E.C. Conf. - 650652, pp. 415-428

DUChaufour, P. - 1965 - Précis de Pédologie, 2." ed., Paris, Masson \& Cie., 481 pp.

LIBBY, W. F. - 1952 - Radiocarbon Dating, 1. ${ }^{\mathrm{a}}$ ed., Chicago University Press

MÜNNICH, K. O. e VOGEL, J. C. - 1958 - Durch Atomexplosionen erzeugter Radiokohlenstoff in der Atmosphäre. Naturwiss. 45: 327-329

MÜNNICH, K. O. e VOGEL, J. C. - 1962 - Investigation of meridional transport in the troposphere by means of carbon-14 measurements. Proc. Symp. Radioactive Dating, I.A.E.A., Vienna, pp. 189-197

NAKHLA, S. A. - 1968 - Étude du comportement du carbone organique dans le sol et étude des podzols à l'aid du carbone-14. Thèse Doct.-ès-Sci., Paris

NYDAL, R. - 1963 - Increase from the most recent series of thermonuclear tests. Nature, 200 p. 212

NYDAL, R. - 1968 - Further Investigation on the Transfer of Radiocarbon in Nature. J. Geophys. Res., 73: 3617-3635

RAFTER, T. A. e FERGUSSON, G. J. - 1957 - The atom bomb effect. Recent increase in the G-14 content of the atmosphere, biosphere and surface waters of the oceans. N.Z. Jl Sci. Tech., B38: 871-883

RAFTER, T. A. e FERGUSSON, G. J. - 1965 - Carbon-14 variations in nature, Part 2 - Increase in ${ }^{14} \mathrm{C}$ activity in the atmosphere of the southern hemisphere from the testing of nuclear weapons. N.Z. JI Sci. Tech., 8: 472-493 
RAFTER, T. A. - 1968 - Carbon-14 variations in nature, Part $3-{ }^{14} \mathrm{C}$ measurements in the South Pacific and Antarctic Oceans. N.Z. Jl Sci. Tech., 11: 551-589

REVELLE, R. e SUESS, H. - 1957 - Carbon dioxide exchange between atmosphere and ocean and the question of an increase of atmospheric $\mathrm{CO}_{2}$ during the past decades. Tellus, 9: 18-27

SCHARPENSEEL, H. W. e KRAUSSE, R. - 1962 - Aminosäurenuntersuchungen an verschiedenen organischen Sedimenten, besonders Grau- und Braunhuminsäurefraktionen verschiedener Bodentypen (einschlieBlich $\mathrm{C}_{14^{-}}$markierter Huminsäuren). Zeitschr. f. Pflanzenernähr, 96 (1): 11-34

SCHAR PENSEEL, H. W. e PIETIG, F. - 1970 - University of Bonn Natural Radiocarbon Measurements III. Radiocarbon, 12 (1): 19-39

SIEFFERMAN, G. Comunicação pessoal

STUIVER, M. - 1961 - Variations in radiocarbon concentration and sunspot activity. J. Geophys. Res., $66(1):$ 273-276

THOMANN, CH. - 1963 - Quelques observations sur l'extraction de l'humus dans le sol: méthode au pyrophosphate de sodium. Cah. Péd. O.R.S.T.O.M., 3: 43-71

VOGEL, J. C. - 1971 - Radiocarbon in nature. Sth Afr. J. Sci., fev, 1971, pp. 32-42 